Monatsschrift f. Geburtshülfe u. Gynäkologie 1922;60:I-IV

\title{
Contents, Vol. 60, 1922
}

\section{Inhaltsverzeichnis.}

Seite

Ansprache von Prof. Dr. W. Zangemeister 1

$\mathrm{A} b$ e r n e $11 \mathrm{y}, \mathrm{C}$, Indikationen zur inneren Untersuchung der Hebammen 243

B e n t h i n, W., Die geburtshilflich-gynäkologische freie Peri tonitis. (Mit spezieller Berücksichtigung der Äther-

therapie) $\quad 171$

Blohmke, A., Mittelohreiterung und Gravidität .... 291

Cohn, Th. , Ein Fall von verkalktem Uterusmyom. Ein

Beitrag zur Klinik der Harnbeschwerden bei Gebär-

muttergeschwülsten 308

Fink, K.. Die praktische BewertungvonNierenafiektionen und

pathologischen Harnbefunden während der Schwanger-

schaft 229

Fuchs, H., Zur Kritik der Ventrofixatio uteri auf Grund

einer Eigenserie von 218 Vesicoventrofixationen . . . 133

Gaydoul, W., und W. Schmitt, Die operative Behand-

lung des Carcinoma colli uteri

Hammerschlag, Über die Aussichten der Infektions-

bekärapfung . -

80

$\mathrm{H}$ e $\mathrm{n}$ k e 1 , M., Die praktische Bewertung des Fiebers unter

der Geburt 58

H e n n i g, Beitrag zur Ätiologie der kongenitalen Dünndarni-

atresie 342

$\mathrm{H}$ enrard , E., Zur Behandlung puerperaler Allgemeininfek-

tionen mit Trypaflavin und Yatren 347

Hofmeier, M., Zur Behandlung der Placenta praevia. . 3

von Klein, C. U., Zur Vagitusfrage. Zwei eigene Be-

obachtungen; gelungene Provokation der Schreiwieder-

holung 154

Lepehne, Zur Kenntnis des Icterus neonatorum . . . 277

Müller, E., und C. Richter, Über Provokationsverfahren

bei Gonorrhoe, insbesondere durch intravenöse Injek-

tionen von Traubenzuckerlösung 355

Naujoks, H., Experimentelle Untersuchungen über die

Wirkung intraperitonealer Ätherinjektionen .... 265

*.

$-\mathrm{IV}-$ 
S $\theta$ it $\theta$

Offermann, W., Zur Diagnose der Zwillingsschwanger-

schaft .259

Riediger, K., Beitrag zur Genitaltuberkulose. .... 225 Rosenstein, P., Über Varizenbildung in der Harnblase

und ihre diagnostische Bedeutung $10 \mathrm{G}$

Rosinski, B., Zur Therapie der Schwangerschaftspyelitis 116 Sachs, E., Über die sogenannte „,intraligamentäre” Blase 88

Schroeder, E., Über Harnfisteloperationen 124

Schütze, Ein Fall von Darmstenose bei Karzinoin der

Flexura sigmoidea, einen Adnextumor vortäuschend.

Darmresektion. Primäre Naht. Dauerheilung .... 130 Unterberger, F. •, Experimentelle

Röntgenschädigung der

Ovarien und ihr Einfluß auf die Nachkommenschaft.

(Hierzu Tafel I) 164

$\mathrm{W}$ a $1 \mathrm{z}, \mathrm{W}$., Über die Bedeutung der intrauterinen Atem-

bewegungen 331

Wüsthoff, H-, Die Frühdiagnose der angeborenen Herz-

fehler 365

Zangemeister, W., und F. Schilling, Über Placenta

cervicalis 15

Ansprache von Geh.-Rat Prof. Dr. A. Martin 373

Bibliographie von Georg Winter 376 